\title{
Morphological Studies on the Suitability of Clerodendrum inermi, Leucophyllum frutescens and Acalypha hispida Shrubs for use in Landscaping under Salinity Conditions
}

\author{
Kathari Lakshmaiah $^{1 *}$, P. Aruna ${ }^{1}$, M. Ganga ${ }^{1}$ and K. Arulmozhiselvan ${ }^{2}$ \\ ${ }^{1}$ Department of Floriculture and Landscape Gardening, Tamil Nadu Agricultural University, \\ Coimbatore, India. ${ }^{2}$ Department of Soil Science, Tamil Nadu Agricultural University, \\ Coimbatore, India \\ *Corresponding author
}

\begin{tabular}{|l|}
\hline Ke y w o r d s \\
Salinity, \\
Morphological \\
parameters, Salt \\
concentrations, \\
$\mathrm{NaCl}$
\end{tabular}

A B S T R A C T
An experiment was conducted at the Botanic Gardens, Department of Floriculture and Landscaping, Horticultural College and Research Institute, Tamil Nadu Agricultural University, Coimbatore during 2015-2016. The present study pertains to the effect of salt stress on three ornamental shrubs of Clerodendrum inerme, Leucophyllum frutescens and Acalypha hispida and their suitability for salinity condition. Ten saline treatments were taken viz., 6, 12, 18, 24, 30, 36, 42, 48, 54dS/m and0.04 dS/m (Control). The treatments were imposed by irrigating the plants with desired amount of $\mathrm{NaCl}$ dissolved in irrigation water. Plants were watered on alternate days $\left(1\right.$ litre plant $\left.{ }^{-1}\right)$ to simulated salinity conditions as seen near coastal belt. The observations were recorded on morphological characters, Clerodendrum and Leucophyllum were tolerant to salinity. Leucophyllum and Clerodendrum experienced a strong reduction in growth and a delay in flowering but no toxicity symptoms or mortality was recorded. These species were found to be moderate $\mathrm{NaCl}$ accumulators. Acalypha was sensitive to salinity, as 50\% of the plants exhibited mortality and the surviving ones experienced a heavy reduction of growth and increased accumulation of $\mathrm{NaCl}$ in the leaves. At higher salt levels, morphological parameters including plant height, root length, number of leaves, leaf thickness, and plant spread were significantly reduced for all the plant species. However, in Leucophyllum and Clerodendrum, $\mathrm{NaCl}$ caused slight decrease only in the morphological parameters as compared with Acalypha.

\section{Introduction}

Among the commercial ornamental plants, Clerodendrum inerme (Verbenaceae) is a much branched, straggling shrub, 1-2 m tall. The plant is tough to sustain periodic trimming well, and hence, is commonly used as a hedge plant in India. It grows well on the beach, tolerating all the salty water sprays. Within India, it is found throughout, particularly near coastal regions. Acalypha hispida, the Chenille plant is a flowering shrub which belongs to the family Euphorbiaceae. Acalypha is the fourth largest genus of the 
Euphorbiaceae family, and contains many plants native to Hawaii and Oceania. The plant is dioecious, and therefore there are distinct male and female members of the species. The female plant bears pistillate flowers which range in colour from purple to bright red, and grow in clusters along catkins. This feature is the primary reason the plant bears the nickname "red-hot cat tail". The pistillates will grow all year long as long as the temperatures are favourable. Leucophyllum frutescens is an evergreen shrub in the figwort family, Scrophulariaceae, native to the state of Texas in the southwestern United States and northern Mexico. The species is commonly referred as Texas sage, Texas Ranger, Texas rain sage, Texas silverleaf, Texas barometerbush, ash-bush, wild lilac, purple sage. Texas Sage is a popular ornamental plant, commonly used for edge in warmer and drier areas. It is available in a variety of cultivars, including 'Green Cloud', 'White Cloud', 'Compacta', 'Convent', and 'Bert-Star'.

Salinity is one of the major environmental factors limiting plant growth and productivity. It is estimated that about one-third of world's cultivated land is affected by salinity (Kaya et al., 2003). Worldwide, more than 800 million hectares of land are salt affected and tolerance to this salinity differs greatly among plant species (Munns and Tester, 2008). In India alone, about 30 million hectares of coastal land is lying barren and uncultivable because of soil affected by salinity. Salt stress in soil or water is one of the major stresses especially in arid and semi-arid regions and can severely limit plant growth and productivity (Allakhverdier et al., 2000). It is a common environmental problem and an important factor limiting crop production, since it is a combined result of the complex interactions among different morphological processes (Singh and Chatrath, 2001; Munns and Tester, 2008).
Of late, one of the major factors in the salt tolerance is believed to be the existence of succulence. Halophytes survive salt concentration equal to or greater than that of seawater and possess physiological mechanisms that maintain lower water potential inside the cell than that in the soil (Munns and Termaat, 1986). In saline environment, controlling the salt concentration of the aerial plant parts by restriction of entry through the roots (is there any role of caspacian layer in roots) and limiting transport to the shoots is an important mechanism allowing plants to survive and grow under salinity (Colmer et al., 2005). The main effect of salinity on glycophytes is reduced growth (Munns and Termaat, 1986) and this reduction has been used in many studies as a measure of resistance to saline conditions (SanchezBlanco et al., 1991). Hence, the objective of this study was morphologically assessing the growth pattern of three popular ornamental shrubs (Leucophyllum frutescence, Acalypha hispida and Clerodendrum inerme) under different salt concentration levels.

\section{Materials and Methods}

The experiment was conducted at the Botanic Gardens, Department of Floriculture and Landscaping, Horticultural College and Research Institute, Tamil Nadu Agricultural University, Coimbatore during 2015-2016.

\section{Soil characteristics}

The media used for growing was pure river sand which was filled in the plot to a depth of $30 \mathrm{~cm}$ from the growth level and. The media was analyzed for physical and chemical properties and the results are presented in Annexure II.

\section{Water composition}

The experimental field was irrigated using potable water during the initial period of plant 
establishment. The properties of the irrigation water were used are given in Annexure III.

List of plant species used for the screening study: The plant species involved in the study and their source is furnished in Table 1.

The selected plant species were screened for tolerance to various levels of salinity stress, based on the observations carried out on growth, morphological, physiological, and biochemical parameters.

\section{Statistical design}

Design: Factorial Completely Randomized Design (FCRD) (Table 2).

Duration of the study: 90 days

\section{Number of replications: 2}

Planting and study period: The cuttings were planted during August, 2015 in earthen pots of $40 \times 30 \mathrm{~cm}$ size, provided with drainage holes at the bottom. The pots were filled with $5 \mathrm{~kg}$ soil containing mixture of sand, soil and FYM in the ratio of $1: 2: 1$. The plants were maintained at optimal conditions by watering, weeding, manuring and plant protection sprays and were allowed to grow until they attained three to four fully mature leaves (90 days). The salinity stress treatments as indicated below were imposed when the plants attained three months of age so as to evaluate the influence of salt stress.

Imposition of salt stress: The stress treatments were imposed by irrigating with the $\mathrm{NaCl}$ dissolved water. Plants were watered at alternate days (1 litre plant ${ }^{-1}$ ) with $\mathrm{NaCl}$ dissolved water to provide respective concentration of EC $(6,12,18,24,30,36,42$, 48, 54dS/m and $0.04 \mathrm{dS} / \mathrm{m}$ (Control) after measuring the moisture content of the soil. For control, set of plants was maintained adjacent to the each of the treatment. The control $\left(\mathrm{T}_{10}\right)$ plant were irrigated with water $(\mathrm{EC}=0.04$ $\mathrm{dS} / \mathrm{m}$ and $6.8 \mathrm{pH}$ ) without any added $\mathrm{NaCl}$. The treatment details are as follows.

\section{Treatment details}

\begin{tabular}{|l|l|}
\hline Treatment Notation & EC $(\mathrm{dS} / \mathrm{m})$ \\
\hline $\mathbf{T}_{\mathbf{1}}$ & 6 \\
\hline $\mathbf{T}_{\mathbf{2}}$ & 12 \\
\hline $\mathbf{T}_{\mathbf{3}}$ & 18 \\
\hline $\mathbf{T}_{\mathbf{4}}$ & 24 \\
\hline $\mathbf{T}_{\mathbf{5}}$ & 30 \\
\hline $\mathbf{T}_{\mathbf{6}}$ & 36 \\
\hline $\mathbf{T}_{\mathbf{7}}$ & 42 \\
\hline $\mathbf{T}_{\mathbf{8}}$ & 48 \\
\hline $\mathbf{T}_{\mathbf{9}}$ & 54 \\
\hline Control- $\mathbf{T}_{\mathbf{1 0}}$ & 0.04 (Siruvani water)* \\
\hline
\end{tabular}

* Siruvani water is from Siruvani river in Coimbatore, which is a major source of drinking water.

\section{Result and Discussion}

Morphological assessment on the growth pattern of three popular ornamental shrubs (Leucophyllum frutescence, Acalypha hispida and Clerodendrum inerme) under different salt concentration levels. The plant height $(\mathrm{cm})$ showed significant difference in the interaction effect between plants and salt levels. The highest plant height was recorded in Clerodendrum inerme $\mathrm{P}_{1} \mathrm{~T}_{10}(49.98 \mathrm{~cm})$, whereas the least plant height was recorded in $\mathrm{P}_{1} \mathrm{~T}_{8}(33.02 \mathrm{~cm})$ followed by Acalypha hispida $\mathrm{P}_{1} \mathrm{~T}_{7}(34.35 \mathrm{~cm})$. When comparing with control $(0.04 \mathrm{dS} / \mathrm{m})$ the least reduction $(21.8 \%)$ in plant height was also observed in Leucophyllum frutescens and the highest reduction $(31.6 \%)$ was observed in Clerodendrum inerme at $\mathrm{T}_{9}$ treatment (Table 3).

The observations on the effect of different concentrations of salinity on the root length of Clerodendrum inerme, Leucophyllum 
frutescens and Acalypha hispida plant spp. on the $90^{\text {th }}$ day after saline treatments are presented in Table 4. Significant differences were observed in the interaction effect between plants and salt levels. The highest root length was recorded in $\mathrm{P}_{1} \mathrm{~T}_{10}(82.92 \mathrm{~cm})$, whereas the lowest root length was recorded in $\mathrm{P}_{3} \mathrm{~T}_{5}(22.15 \mathrm{~cm})$ followed by $\mathrm{P}_{3} \mathrm{~T}_{4}(24.20$ $\mathrm{cm})$. The root length of Clerodendrum inerme followed a decreasing trend with increasing salinity, except for $\mathrm{T}_{9}$ which had higher root length. In Leucophyllum frutescens $\mathrm{T}_{3}, \mathrm{~T}_{4}$ and $\mathrm{T}_{5}$ was on par with each other. In Acalypha hispida the root length gradually decreased from $T_{1}$ to $T_{5}$ and the remaining treatments showed absolute mortality after $60^{\text {th }}$ day onwards. A stimulation of growth in response to moderate levels of $\mathrm{NaCl}$ salinity has been reported in several halophytes. Sesuvium portulacastrum survived up to $900 \mathrm{mM} \mathrm{NaCl}$, but produced favorauble growth at $600 \mathrm{mM}$ $\mathrm{NaCl}$ (Venkatesalu et al., 1994). Salicornia europaea and Arthocnemum australiasicum survived double the strength of seawater (McMillan, 1974). A positive growth response to moderate salinity has been reported in the mangrove species, Avicennia marina (Downton, 1982; Clough, 1984) and Aegiceras corniculatum (Ball and Forquhar, 1984).

Growth related traits are used as harbingers for salinity tolerance of plant spp. In the present study, significant differences were observed in the interaction effect between plant species and salt levels in Root/Shoot ratio (Table 5). The maximum Root/Shoot ratio (1.82) was recorded in $\mathrm{P}_{1} \mathrm{~T}_{1}(6 \mathrm{dS} / \mathrm{m})$, which was followed by $\mathrm{P}_{1} \mathrm{~T}_{9}$ (1.77) whereas the minimum Root/Shoot ratio was recorded in $\mathrm{P}_{3} \mathrm{~T}_{5}(0.58)$ followed by $\mathrm{P}_{3} \mathrm{~T}_{4}(0.62)$ and $\mathrm{P}_{3} \mathrm{~T}_{2}(0.65)$ and $\mathrm{P}_{3} \mathrm{~T}_{3}(0.70)$. The shoot: root of Clerodendrum inerme, Leucophyllum frutescens and Acalypha hispida plant spp. significantly decreased under salinity stress compared with that of the control. In
Clerodendrum and Leucophyllum plants, increased shoot : root ratio were recorded, while Acalypha plants recorded the lowest shoot : root ratio. A lower shoot: root seemed to be related to salt tolerance (Dudeck et al., 1983; Dudeck and Peacock, 1985) but can also be a genetic trait.

The observations on the effect of different concentrations of $\mathrm{NaCl}$ salinity on the leaf count in Clerodendrum inerme, Leucophyllum frutescens and Acalypha hispida plant species. on the $90^{\text {th }}$ day after saline water treatment are presented in Table 6. Regarding interaction effect in Leucophyllum frutescens andClerodendrum inerme the number of leaves reduced significantly from lower salt level to higher salt $(54 \mathrm{dS} / \mathrm{m})$ but in Acalypha hispida the plant can survive up to only 30 $\mathrm{dS} / \mathrm{m}$, where the maximum reduction in number of leaves were observed. The interaction between the plant species and salt level was significantly different. The maximum number of leaves were recorded in $\mathrm{P}_{2} \mathrm{~T}_{10}$ (78.76) and the minimum number of leaves were recorded in $\mathrm{P}_{3} \mathrm{~T}_{5}$.

The number of leaves in Clerodendrum inerme, and Leucophyllum frutescens gradually decreased from $\mathrm{T}_{1}$ to $\mathrm{T}_{9}$. The decline in leaf number at high concentrations was due to the leaf fall because of ageing. Salinity has been shown to be one of the external factors that influence the process of senescence and the consequent shedding of leaves (Pool et al., 1975). The number of leaves reduced only at the highest salt concentration but the number of dead leaves increased with salinity as a means of protecting the young growing leaves to toxic levels of the salts as well as offloading the plants of excess salts (Wahome, 2001). Fresh weights of leaf, stem and root increased with increasing salinity up to the optimum concentrations. 
The observations on the effect of different concentrations of $\mathrm{NaCl}$ salinity on the leaf thickness of Clerodendrum inerme, Leucophyllum frutescens and Acalypha hispida plant spp. on the $60^{\text {th }}$ day after saline water treatments are presented in Table 7. Significant differences were observed in the interaction effect between plant species and salt levels. The maximum leaf thickness was recorded in $\mathrm{P}_{1} \mathrm{~T}_{9}(4 \mathrm{~mm})$ followed by $\mathrm{P}_{1} \mathrm{~T}_{8}$ $(3.45 \mathrm{~mm})$, whereas the minimum leaf thickness was recorded in $\mathrm{P}_{3} \mathrm{~T}_{7}(1.40 \mathrm{~mm})$ followed by $\mathrm{P}_{3} \mathrm{~T}_{6}(1.63 \mathrm{~mm})$. The thickness of leaves in Clerodendrum inerme gradually increased at $\mathrm{T}_{1}$ to $\mathrm{T}_{9}$. Leucophyllum frutescens and Acalypha hispida were on par with each other.

Table.1 List of plant species used for the screening study

\begin{tabular}{|l|l|l|}
\hline Common name & Botanical name & Source \\
\hline Glory bower & Clerodendrum inerme & Botanic Gardens, TNAU \\
\hline Purple sage & Leucophyllum frutescens & Botanic Gardens, TNAU \\
\hline Red-hot cat tail & Acalypha hispida & Botanic Gardens, TNAU \\
\hline
\end{tabular}

Table.2 Factorial Completely Randomized Design (FCRD)

\begin{tabular}{|c|c|c|c|}
\hline S. & FACTOR & \multicolumn{2}{|c|}{ SPECIES / LEVELS IN FACTOR } \\
\hline 1 & $\begin{array}{l}\text { Factor } 1 \quad- \\
\text { Plant } \\
\text { species }\end{array}$ & $\begin{array}{l}\mathbf{P}_{1}-\text { Clerodendr } \\
\mathbf{P}_{2}-\text { Leucophyl } \\
\mathbf{P}_{3}-\text { Acalypha }\end{array}$ & $\begin{array}{l}\text { inerme } \\
\text { f frutescens } \\
\text { ida }\end{array}$ \\
\hline 2 & $\begin{array}{l}\text { Factor 2 - } \\
\text { Salinity } \\
\text { Levels }\end{array}$ & $\begin{array}{l}S_{1}-6 d S S ~ m^{-1} \\
S_{2}-12 d^{-1} m^{-1} \\
S_{3}-18 d S m^{-1} \\
S_{4}-24 d S m^{-1} \\
S_{5}-30 d S m^{-1}\end{array}$ & $\begin{array}{l}S_{6}-36 \mathrm{dS} \mathrm{m}^{-1} \\
S_{7}-42 \mathrm{dS} \mathrm{m}^{-1} \\
S_{8}-48 \mathrm{dS} \mathrm{m}^{-1} \\
S_{9}-54 \mathrm{dS}^{-1} \\
S_{10}-0.04 \mathrm{dS} \mathrm{m}^{-1} \text { (Control) }\end{array}$ \\
\hline
\end{tabular}

Plate.1 Field view

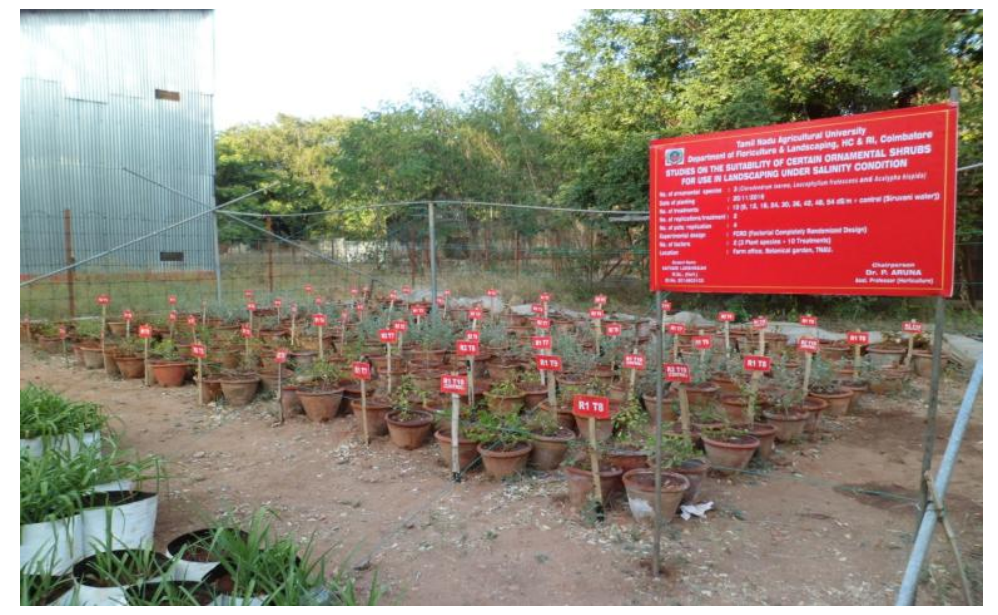


Int.J.Curr.Microbiol.App.Sci (2018) 7(12): 1385-1397

Table.3 Effect of salinity on plant height $(\mathrm{cm})$ of Clerodendrum inerme, Leucophyllum frutescens and Acalypha hispida

\begin{tabular}{|c|c|c|c|c|c|c|c|c|c|c|c|}
\hline \multirow[t]{2}{*}{ Treatments } & \multicolumn{11}{|c|}{ Plant height $(\mathrm{cm})$} \\
\hline & $\mathbf{T}_{1}$ & $\mathbf{T}_{2}$ & $\mathbf{T}_{\mathbf{3}}$ & $\mathbf{T}_{4}$ & $\mathbf{T}_{5}$ & $\mathbf{T}_{6}$ & $\mathbf{T}_{7}$ & $\mathbf{T}_{8}$ & $\mathbf{T}_{9}$ & $\mathbf{T}_{10}$ & Mean \\
\hline $\mathrm{P}_{1}$ & 45.00 & 41.44 & 42.02 & 39.76 & 36.45 & 35.06 & 34.35 & 33.02 & 34.19 & 49.98 & 39.13 \\
\hline $\mathrm{P}_{2}$ & 44.59 & 41.44 & 42.74 & 42.43 & 37.83 & 36.84 & 36.33 & 34.59 & 36.05 & 46.10 & 39.89 \\
\hline $\mathrm{P}_{3}$ & 44.79 & 41.83 & 41.10 & 40.27 & 37.43 & 0.10 & 0.09 & 0.09 & 0.10 & 46.10 & 25.19 \\
\hline Mean & 44.79 & 41.57 & 41.95 & 40.82 & 37.24 & 24.00 & 23.59 & 22.57 & 23.45 & 47.39 & 34.74 \\
\hline Interactions & \multicolumn{4}{|c|}{$\mathbf{P}$} & \multicolumn{4}{|c|}{$\mathbf{T}$} & \multicolumn{3}{|c|}{ PX T } \\
\hline $\mathrm{SE}(\mathrm{d})$ & \multicolumn{4}{|c|}{0.37} & \multicolumn{4}{|c|}{0.69} & \multicolumn{3}{|c|}{1.19} \\
\hline $\mathrm{CD}(\mathrm{P}=0.05)$ & \multicolumn{4}{|c|}{$0.77 * *$} & \multicolumn{4}{|c|}{$1.41 * *$} & \multicolumn{3}{|c|}{$2.44 * *$} \\
\hline \multicolumn{3}{|c|}{ Plant species } & \multicolumn{4}{|c|}{ Salt concentrations $(\mathrm{dS} / \mathrm{m})$} & \multicolumn{5}{|c|}{ **Highly significant } \\
\hline \multicolumn{3}{|c|}{$\mathbf{P}_{1^{-}}$Clerodendrum inerme } & $\mathrm{T}_{1}-6$ & $\mathrm{~T}_{4}-24$ & $\mathrm{~T}_{7}-42$ & \multirow[t]{3}{*}{$\begin{array}{c}\mathrm{T}_{10}-0.04 \\
\text { (control) }\end{array}$} & & & & & \\
\hline \multicolumn{3}{|c|}{$\mathbf{P}_{2}-$ Leucophyllum frutescens } & $\mathrm{T}_{2}-12$ & $\mathrm{~T}_{5}-30$ & $\mathrm{~T}_{8}-48$ & & & & & & \\
\hline \multicolumn{3}{|c|}{$\mathbf{P}_{3^{-}}$Acalypha hispida } & $\mathrm{T}_{3}-18$ & $\mathrm{~T}_{6}-36$ & $\mathrm{~T}_{9}-54$ & & & & & & \\
\hline
\end{tabular}


Table.4 Effect of salinity on Plant root length (cm) of Clerodendrum inerme, Leucophyllum frutescens and Acalypha hispida

\begin{tabular}{|c|c|c|c|c|c|c|c|c|c|c|c|}
\hline \multirow[t]{2}{*}{ Treatments } & \multicolumn{11}{|c|}{ Plant root length $(\mathrm{cm})$} \\
\hline & $\mathbf{T}_{1}$ & $\mathbf{T}_{2}$ & $\mathbf{T}_{\mathbf{3}}$ & $\mathbf{T}_{4}$ & $\mathbf{T}_{5}$ & $\mathbf{T}_{6}$ & $\mathbf{T}_{7}$ & $\mathbf{T}_{8}$ & $\mathbf{T}_{9}$ & $\mathbf{T}_{10}$ & Mean \\
\hline $\mathrm{P}_{1}$ & 79.95 & 71.05 & 70.79 & 66.43 & 61.64 & 58.51 & 58.01 & 56.84 & 58.83 & 82.92 & 66.50 \\
\hline $\mathrm{P}_{2}$ & 68.88 & 63.08 & 65.48 & 65.48 & 64.68 & 61.05 & 58.90 & 57.91 & 58.50 & 71.40 & 63.54 \\
\hline $\mathrm{P}_{3}$ & 31.47 & 28.37 & 28.35 & 24.20 & 22.15 & 0.10 & 0.09 & 0.09 & 0.10 & 37.74 & 17.27 \\
\hline Mean & 60.10 & 54.17 & 54.87 & 52.04 & 49.49 & 39.89 & 39.00 & 38.28 & 39.14 & 64.02 & 49.10 \\
\hline Interactions & \multicolumn{4}{|c|}{$\mathbf{P}$} & \multicolumn{4}{|c|}{$\mathbf{T}$} & \multicolumn{3}{|c|}{ P X T } \\
\hline $\mathrm{SE}(\mathrm{d})$ & \multicolumn{4}{|c|}{0.54} & \multicolumn{4}{|c|}{0.99} & \multicolumn{3}{|c|}{1.72} \\
\hline $\mathrm{CD}(\mathrm{P}=0.05)$ & \multicolumn{4}{|c|}{$1.11 * *$} & \multicolumn{4}{|c|}{$2.03 * *$} & \multicolumn{3}{|c|}{$3.52 * *$} \\
\hline \multicolumn{3}{|c|}{ Plant species } & \multicolumn{4}{|c|}{ Salt concentrations $(\mathrm{dS} / \mathrm{m})$} & \multicolumn{5}{|c|}{ **Highly significant } \\
\hline \multicolumn{3}{|c|}{$\mathbf{P}_{1-}$ Clerodendrum inerme } & $\mathrm{T}_{1}-6$ & $\mathrm{~T}_{4}-24$ & $\mathrm{~T}_{7}-42$ & \multirow[t]{3}{*}{$\begin{array}{l}\mathrm{T}_{10}-0.04 \\
\text { (Control) }\end{array}$} & & & & & \\
\hline \multicolumn{3}{|c|}{$\mathrm{P}_{2}-$ Leucophyllum frutescens } & $\mathrm{T}_{2}-12$ & $\mathrm{~T}_{5}-30$ & $\mathrm{~T}_{8}-48$ & & & & & & \\
\hline \multicolumn{3}{|c|}{$\mathbf{P}_{3-}$ Acalypha hispida } & $\mathrm{T}_{3}-18$ & $\mathrm{~T}_{6}-36$ & $\mathrm{~T}_{9}-54$ & & & & & & \\
\hline
\end{tabular}


Int.J.Curr.Microbiol.App.Sci (2018) 7(12): 1385-1397

Table.5 Effect of salinity on Root shoot ratio of Clerodendrum inerme, Leucophyllum frutescens and Acalypha hispida

\begin{tabular}{|c|c|c|c|c|c|c|c|c|c|c|c|}
\hline \multirow[t]{2}{*}{ Treatments } & \multicolumn{11}{|c|}{ Root shoot ratio } \\
\hline & $\mathbf{T}_{1}$ & $\mathbf{T}_{2}$ & $\mathbf{T}_{\mathbf{3}}$ & $\mathbf{T}_{4}$ & $\mathbf{T}_{5}$ & $\mathbf{T}_{6}$ & $\mathbf{T}_{7}$ & $\mathbf{T}_{8}$ & $\mathbf{T}_{9}$ & $\mathbf{T}_{10}$ & Mean \\
\hline $\mathrm{P}_{1}$ & 1.82 & 1.67 & 1.72 & 1.72 & 1.66 & 1.64 & 1.67 & 1.69 & 1.77 & 1.69 & 1.71 \\
\hline $\mathrm{P}_{2}$ & 1.58 & 1.49 & 1.56 & 1.57 & 1.58 & 1.57 & 1.58 & 1.60 & 1.67 & 1.58 & 1.58 \\
\hline $\mathrm{P}_{3}$ & 0.72 & 0.65 & 0.70 & 0.62 & 0.58 & 0.98 & 0.99 & 0.98 & 1.03 & 0.82 & 0.81 \\
\hline Mean & 1.38 & 1.27 & 1.33 & 1.30 & 1.27 & 1.40 & 1.41 & 1.42 & 1.49 & 1.36 & 1.36 \\
\hline Interactions & \multicolumn{4}{|c|}{$\mathbf{P}$} & \multicolumn{4}{|c|}{$\mathbf{T}$} & \multicolumn{3}{|c|}{ P X T } \\
\hline $\mathrm{SE}(\mathrm{d})$ & \multicolumn{4}{|c|}{0.014} & \multicolumn{4}{|c|}{0.025} & \multicolumn{3}{|c|}{0.044} \\
\hline $\mathrm{CD}(\mathrm{P}=0.05)$ & \multicolumn{4}{|c|}{$0.022 * *$} & \multicolumn{4}{|c|}{$0.052 * *$} & \multicolumn{3}{|c|}{$0.091 * *$} \\
\hline \multicolumn{3}{|c|}{ Plant species } & \multicolumn{4}{|c|}{ Salt concentrations $(\mathrm{dS} / \mathrm{m})$} & \multicolumn{5}{|c|}{ **Highly significant } \\
\hline \multicolumn{3}{|c|}{$\mathbf{P}_{1^{-}}$Clerodendrum inerme } & $\mathrm{T}_{1}-6$ & $\mathrm{~T}_{4}-24$ & $\mathrm{~T}_{7}-42$ & \multirow[t]{3}{*}{$\begin{array}{l}\mathrm{T}_{10}-0.04 \\
\text { (Control) }\end{array}$} & & & & & \\
\hline \multicolumn{3}{|c|}{$\mathbf{P}_{2}-$ Leucophyllum frutescens } & $\mathrm{T}_{2}-12$ & $\mathrm{~T}_{5}-30$ & $\mathrm{~T}_{8}-48$ & & & & & & \\
\hline \multicolumn{3}{|c|}{$\mathbf{P}_{3^{-}}$Acalypha hispida } & $\mathrm{T}_{3}-18$ & $\mathrm{~T}_{6}-36$ & $\mathrm{~T}_{9}-54$ & & & & & & \\
\hline
\end{tabular}


Int.J.Curr.Microbiol.App.Sci (2018) 7(12): 1385-1397

Table.6 Effect of salinity on number of leaves of Clerodendrum inerme, Leucophyllum frutescens and Acalypha hispida

\begin{tabular}{|c|c|c|c|c|c|c|c|c|c|c|c|}
\hline \multirow[t]{2}{*}{ Treatments } & \multicolumn{11}{|c|}{ No of leaves } \\
\hline & $\mathbf{T}_{1}$ & $\mathbf{T}_{2}$ & $\mathbf{T}_{\mathbf{3}}$ & $\mathbf{T}_{4}$ & $\mathbf{T}_{5}$ & $\mathbf{T}_{6}$ & $\mathbf{T}_{7}$ & $\mathbf{T}_{8}$ & $\mathbf{T}_{9}$ & $\mathbf{T}_{10}$ & Mean \\
\hline $\mathrm{P}_{1}$ & 42.14 & 40.00 & 38.39 & 38.10 & 39.48 & 38.60 & 37.32 & 36.28 & 35.72 & 43.09 & 38.91 \\
\hline $\mathrm{P}_{2}$ & 72.01 & 70.50 & 70.45 & 68.54 & 65.87 & 64.58 & 62.77 & 62.94 & 60.97 & 78.76 & 67.74 \\
\hline $\mathrm{P}_{3}$ & 22.25 & 17.66 & 11.84 & 8.98 & 8.00 & - & - & - & - & 28.42 & 16.19 \\
\hline Mean & 45.47 & 42.72 & 40.23 & 38.54 & 37.78 & 51.59 & 50.05 & 49.61 & 48.35 & 50.09 & 40.95 \\
\hline Interactions & \multicolumn{4}{|c|}{$\mathbf{P}$} & \multicolumn{4}{|c|}{$\mathbf{T}$} & \multicolumn{3}{|c|}{ P X T } \\
\hline $\mathrm{SE}(\mathrm{d})$ & \multicolumn{4}{|c|}{0.20} & \multicolumn{4}{|c|}{0.37} & \multicolumn{3}{|c|}{0.64} \\
\hline $\mathrm{CD}(\mathrm{P}=0.05)$ & \multicolumn{4}{|c|}{0.41} & \multicolumn{4}{|c|}{0.75} & \multicolumn{3}{|c|}{$1.30 * *$} \\
\hline \multicolumn{3}{|c|}{ Plant species } & \multicolumn{4}{|c|}{ Salt concentrations $(\mathrm{dS} / \mathrm{m})$} & \multicolumn{4}{|c|}{ **Highly significant } & \\
\hline \multicolumn{3}{|c|}{$\mathbf{P}_{1^{-}}$Clerodendrum inerme } & $\mathrm{T}_{1}-6$ & $\mathrm{~T}_{4}-24$ & $\mathrm{~T}_{7}-42$ & \multirow[t]{3}{*}{$\begin{array}{l}\mathrm{T}_{10}-0.04 \\
\text { (control) }\end{array}$} & & & & & \\
\hline \multicolumn{3}{|c|}{$\mathbf{P}_{2}$ - Leucophyllum frutescens } & $\mathrm{T}_{2}-12$ & $\mathrm{~T}_{5}-30$ & $\mathrm{~T}_{8}-48$ & & & & & & \\
\hline \multicolumn{3}{|c|}{$\mathbf{P}_{3}-$ Acalypha hispida } & $\mathrm{T}_{3}-18$ & $\mathrm{~T}_{6}-36$ & $\mathrm{~T}_{9}-54$ & & & & & & \\
\hline
\end{tabular}


Int.J.Curr.Microbiol.App.Sci (2018) 7(12): 1385-1397

Table.7 Effect of salinity on Leaf thickness (mm) of Clerodendrum inerme, Leucophyllum frutescens and Acalypha hispida

\begin{tabular}{|c|c|c|c|c|c|c|c|c|c|c|c|}
\hline \multirow[t]{2}{*}{ Treatments } & \multicolumn{11}{|c|}{ Leaf thickness (mm) } \\
\hline & $\mathbf{T}_{1}$ & $\mathbf{T}_{2}$ & $\mathbf{T}_{\mathbf{3}}$ & $\mathbf{T}_{4}$ & $\mathbf{T}_{5}$ & $\mathbf{T}_{6}$ & $\mathbf{T}_{7}$ & $\mathbf{T}_{8}$ & $\mathbf{T}_{9}$ & $\mathbf{T}_{\mathbf{1 0}}$ & Mean \\
\hline $\mathrm{P}_{1}$ & 2.34 & 2.32 & 2.63 & 2.82 & 2.87 & 2.99 & 3.19 & 3.45 & 4.00 & 2.27 & 2.89 \\
\hline $\mathrm{P}_{2}$ & 2.22 & 2.18 & 2.31 & 2.36 & 2.27 & 2.32 & 2.35 & 2.41 & 2.61 & 2.27 & 2.33 \\
\hline $\mathrm{P}_{3}$ & 1.84 & 1.82 & 1.70 & 1.73 & 1.78 & 1.63 & 1.40 & 1.82 & 1.97 & 1.87 & 1.76 \\
\hline Mean & 2.13 & 2.11 & 2.21 & 2.30 & 2.31 & 2.31 & 2.31 & 2.56 & 2.86 & 2.14 & 2.32 \\
\hline Interactions & \multicolumn{4}{|c|}{$\mathbf{P}$} & \multicolumn{4}{|c|}{$\mathbf{T}$} & \multicolumn{3}{|c|}{ P X T } \\
\hline $\mathrm{SE}(\mathrm{d})$ & \multicolumn{4}{|c|}{0.02} & \multicolumn{4}{|c|}{0.04} & \multicolumn{3}{|c|}{0.07} \\
\hline $\mathrm{CD}(\mathrm{P}=0.05)$ & \multicolumn{4}{|c|}{$0.04 * *$} & \multicolumn{4}{|c|}{$0.08 * *$} & \multicolumn{3}{|c|}{$0.15 * *$} \\
\hline \multicolumn{3}{|c|}{ Plant species } & \multicolumn{4}{|c|}{ Salt concentrations $(\mathrm{dS} / \mathrm{m})$} & \multicolumn{5}{|c|}{ **Highly significant } \\
\hline \multicolumn{3}{|c|}{$\mathbf{P}_{1^{-}}$Clerodendrum inerme } & $\mathrm{T}_{1}-6$ & $\mathrm{~T}_{4}-24$ & $\mathrm{~T}_{7}-42$ & \multirow[t]{3}{*}{$\begin{array}{l}\mathrm{T}_{10}-0.04 \\
\text { (Control) }\end{array}$} & & & & & \\
\hline \multicolumn{3}{|c|}{$\mathbf{P}_{2}-$ Leucophyllum frutescens } & $\mathrm{T}_{2}-12$ & $\mathrm{~T}_{5}-30$ & $\mathrm{~T}_{8}-48$ & & & & & & \\
\hline \multicolumn{3}{|c|}{$\mathbf{P}_{3^{-}}$Acalypha hispida } & $\mathrm{T}_{3}-18$ & $\mathrm{~T}_{6}-36$ & $\mathrm{~T}_{9}-54$ & & & & & & \\
\hline
\end{tabular}


Table.8 Effect of salinity on Plant spread $\left(\mathrm{cm}^{2}\right)$ of Clerodendrum inerme, Leucophyllum frutescens and Acalypha hispida

\begin{tabular}{|c|c|c|c|c|c|c|c|c|c|c|c|}
\hline \multirow[t]{2}{*}{ Treatments } & \multicolumn{11}{|c|}{ Plant spread $\left(\mathrm{cm}^{2}\right)$} \\
\hline & $\mathbf{T}_{1}$ & $\mathbf{T}_{2}$ & $\mathbf{T}_{3}$ & $\mathbf{T}_{4}$ & $\mathbf{T}_{5}$ & $\mathbf{T}_{6}$ & $\mathbf{T}_{7}$ & $\mathbf{T}_{8}$ & $\mathbf{T}_{9}$ & $\mathbf{T}_{10}$ & Mean \\
\hline $\mathrm{P}_{1}$ & 782.00 & 716.00 & 702.00 & 682.00 & 636.00 & 610.00 & 591.00 & 536.00 & 510.00 & 800.00 & 656.50 \\
\hline $\mathrm{P}_{2}$ & 984.00 & 935.00 & 905.00 & 897.00 & 874.00 & 852.00 & 728.00 & 638.00 & 552.00 & 1036.00 & 840.10 \\
\hline $\mathrm{P}_{3}$ & 303.00 & 288.00 & 243.00 & 240.00 & 209.00 & 180.00 & 136.00 & 122.00 & 107.00 & 320.00 & 214.80 \\
\hline Mean & 689.67 & 646.33 & 616.67 & 606.33 & 573.00 & 547.33 & 485.00 & 432.00 & 389.67 & 718.67 & 570.47 \\
\hline Interactions & \multicolumn{4}{|c|}{$\mathbf{P}$} & \multicolumn{4}{|c|}{$\mathbf{T}$} & \multicolumn{3}{|c|}{$\mathbf{P X} \mathbf{T}$} \\
\hline $\mathrm{SE}(\mathrm{d})$ & \multicolumn{4}{|c|}{6.35} & \multicolumn{4}{|c|}{11.60} & \multicolumn{3}{|c|}{20.10} \\
\hline $\mathrm{CD}(\mathrm{P}=0.05)$ & \multicolumn{4}{|c|}{$12.98 * *$} & \multicolumn{4}{|c|}{$23.70 * *$} & \multicolumn{3}{|c|}{$41.06 * *$} \\
\hline \multicolumn{3}{|c|}{ Plant species } & \multicolumn{4}{|c|}{ Salt concentrations $(\mathrm{dS} / \mathrm{m})$} & \multicolumn{5}{|c|}{ **Highly significant } \\
\hline \multicolumn{3}{|c|}{$\mathbf{P}_{1^{-}}$Clerodendrum inerme } & $\mathrm{T}_{1}-6$ & $\mathrm{~T}_{4}-24$ & $\mathrm{~T}_{7}-42$ & \multirow[t]{3}{*}{$\begin{array}{l}\mathrm{T}_{10}-0.04 \\
\text { (Control) }\end{array}$} & & & & & \\
\hline \multicolumn{3}{|c|}{$\mathbf{P}_{2}$ - Leucophyllum frutescens } & $\mathrm{T}_{2}-12$ & $\mathrm{~T}_{5}-30$ & $\mathrm{~T}_{8}-48$ & & & & & & \\
\hline \multicolumn{3}{|c|}{$\mathbf{P}_{3^{-}}$Acalypha hispida } & $\mathrm{T}_{3}-18$ & $T_{6}-36$ & $\mathrm{~T}_{9}-54$ & & & & & & \\
\hline
\end{tabular}


The increase in fresh weight of the leaf tissue can be attributed to the increase in leaf thickness (Clipson, 1987) and the accumulation of ions and water in the tissues (Khan et al., 2005; Lee et al., 2005). This phenomenon is usually correlated with the accumulation of $\mathrm{Na}^{+}$and $\mathrm{Cl}^{-}$ions in leaf tissue (Karakas et al., 2000) and Aktas et al., (2006). The leaf thickness and midrib thickness recorded under different ecosystems were showing significant differences among them.

The seashore ecotype recorded highest leaf thickness than backwater ecotype and inland ecotype. The common response of plant towards the high salinity is thickening of leaf that can reserve abundant water. Increase in the leaf thickness as salinity response was reported by Ibrahim et al., (1991) and Sobrado and Ewe, (2006). Sobrado and Ewe, (2006) concluded that Lumnitzera racemosa increases leaf thickness and water content when stressed with high salinity.

The plant spread for both Clerodendrum inerme, and Leucophyllum frutescens was decreased as the $\mathrm{NaCl}$ salinity level increased, However, Acalypha hispida showed very less spreading nature (Table 8). Significant differences were observed in the interaction effect between plants and salt levels. The highest plant spread was recorded in $\mathrm{P}_{2} \mathrm{~T}_{10}$ $\left(1036.0 \mathrm{~cm}^{2}\right)$, whereas the least plant spread was recorded in $\mathrm{P}_{3} \mathrm{~T}_{9}\left(107.0 \mathrm{~cm}^{2}\right)$ followed by $\mathrm{P}_{3} \mathrm{~T}_{8}\left(122.0 \mathrm{~cm}^{2}\right)$. This is an indication of the higher salinity tolerance of Clerodendrum inerme, and Leucophyllum frutescens compared with Acalypha hispida. Higher salinity causes salt stress injury on the canopy.

Based on the overall performance for morphological parameters, it can be concluded that salinity tolerance level and of plant species for saline conditions is in order of Leucophyllum frutescens >Clerodendrum inerme> Acalypha hispida. Hence, Leucophyllum frutescens and Clerodendrum inerme; may be recommended for saline situations such as beach resorts, coastal areas, etc.

\section{References}

Aktas, H., Abak, K. and I. Cakmak,. 2006. Genotypic variation in the response of pepper to salinity. Scientia Hortic., 110: 260-266.

Allakhaverdiev S. I., A. Skamoto, Y. Nishiyama, M. Inaba, N. Murata. 2000. Ionic and osmotic effects of $\mathrm{NaCl}$ induced inactivation of photosystems I and II in Synechococcus sp. Plant Physiol., 123: 1047-1056.

Ball, M. C. and G. D. Farquhar, 1984.Photosynthetic and stomatal responses of two mangrove species, Aegicerascorniculatum and Avicennia marina to long term salinity and humidity conditions.Plant Physiol., 62: 889-893.

Clipson, N. J. W. 1987. Salt tolerance in the halophyte Suaeda maritima L. Dum. Growth, ion and water relations and gas exchange in response to altered salinity. J. Exp. Bot., 38: 1996-2004.

Clough, B. F. 1984. Growth and salt balance of the mangroves Avicennia marina (Forsk). Vierh and Rhizophorastylosa Griff. In relation to salinity. Aust. J. Plant Physiol., 11: 419-430.

Colmer, T. D., Munns, R. and Flowers, T. J. 2005. Improving salt tolerance of wheat and barley: future prospects, Aust. J. of Exp. Agri. 45: 1425-1443.

Downton, W. J. S. 1982. Growth and osmotic relations of the mangrove Avicennia marina as influenced by salinity. Aust. J. Biol. Sci., pp. 519-528.

Dudeck, A. E. and Peacock. 1985. Effect of salinity on seashore of Paspalum 
turfgrasses. Agro. J., 77: 47-57.

Dudeck,A. E., S. Singh, E. C. Giordano, T.A. Nell and D.B. McConnel. 1983. Effect of sodium chloride on Cynodon turf grasses. Agron. J., 75: 927-930.

Ibrahim, K., J. Collins and H. Collin. 1991. Effects of salinity on growth and ionic composition of Coleus blumei and Salvia splendens. J Hort. Sci., (United Kingdom).

Karakas, B., Lo Bianco, R. and Rieger, M. 2000. Association of marginal leaf scorch with sodium accumulation in salt-stressed peach. Hort. Sci., 35: 8384.

Kaya, C., Higgs, D., Ince, F., Amador, B. M., Cakir, A. and Sakar, E. 2003. Ameliorative effects of potassium phosphate on salt-stressed pepper and cucumber. J. Plant Nutr.,26: 807-820.

Khan, M.A., I.A. Ungar and A.M. Showalter, 2005. Salt stimulation and tolerance in an inter tidal stem - succulent halophyte. J. Plant Nutr., 28: 13651374.

Lee, G., V. Carrow and A. Duncan, 2005. Growth and water relation responses to salinity stress in halophytic sea shore Paspalum ecotypes, Sci. Hort., 104: 221-236.

McMillan, C. 1974. Salt tolerance of mangroves and submerged aquatic plants. Reimold, R. J. and W. H. Queen (eds.). Pp. 379-390. Academic press, New York.

Munns, R. and A. Termaat, (1986). Whole plant response to salinity, Aust. J. of Plant Physio., 13: 143-160.

Munns, R. and M. Tester. 2008. Mechanisms of salinity tolerance. Annu Rev Plant Biol., 59:651-681.

Pool, D. J., A. E. Lugo and S. C. Snedaker. 1975. Litter production in mangrove forest of Southern Florida and Puerto Rico. In: Proc. Int. Symp. Biol. and Management of mangroves. Walsh, G.E. S.C. Snedaker and H.J. Teas. (eds.), pp. 213-237. Int. Food. Agri. Sci., Univ. Florida, Gainesville.

Sanchez-Blanco, M. J., Bolaroan, M. C., J. J., Alarcoan, A Torrecillas. 1991. Salinity effects on water relations in Lycopersicon esculentum and its wild salt-tolerant relative species. Physiol. Plant. 83: 269 - 274.

Singh, K. N. and R. Chatrath M.P. Reynalds, L. Ortiz-Monasterio and A. McNab, 2001. Salinity tolerance in application of physiology in Wheat Breeding. CIMMYT. Mexico, 101-110

Sobrado, M. and S. M. Ewe. 2006. Ecophysiological characteristics of Avicennia germinans and Laguncularia racemosa coexisting in a scrub mangrove forest at the Indian River Lagoon, Florida. Trees. 20:679-687.

Venkatesalu, V., R. Rajkumar and K. P. Chellappan, 1994. Growth and mineral distribution of Sesuvium portulacastrum L. a salt marsh halophyte, under sodium chloride stress. Common. Soil Sci., Plant Anal., 25: 2797-2805.

Wahome, P. K. 2001. Mechanism of salt stress tolerance in two rose root stocks: Rosa chinensis Major and Rosa robiginosa. Sci. Hort., 87: 207-216.

\section{How to cite this article:}

Kathari Lakshmaiah, P. Aruna, M. Ganga and Arulmozhiselvan, K. 2018. Morphological Studies on the Suitability of Clerodendrum inermi, Leucophyllum frutescens and Acalypha hispida Shrubs for use in Landscaping under Salinity Conditions. Int.J.Curr.Microbiol.App.Sci. 7(12): 1385-1397. doi: https://doi.org/10.20546/ijcmas.2018.712.166 